\title{
THE DISCRIMINANT AND THE DETERMINANT OF A HYPERSURFACE OF EVEN DIMENSION
}

\author{
TAKEShi SAITO
}

\begin{abstract}
For a smooth hypersurface of even dimension, the quadratic character of the absolute Galois group defined by the determinant of the $\ell$-adic cohomology of middle dimension is computed via the square root of the discriminant of a defining polynomial of the hypersurface.
\end{abstract}

Let $k$ be a field, $\bar{k}$ an algebraic closure of $k$ and $k_{s}$ the maximal separable extension of $k$ contained in $\bar{k}$. Let $\Gamma_{k}=\operatorname{Gal}\left(k_{s} / k\right)=\operatorname{Aut}_{k}(\bar{k})$.

Let $X$ be a proper smooth variety of even dimension $n$ over $k$. If $\ell$ is a prime number which is invertible in $k$, the $\ell$-adic cohomology $V=H^{n}\left(X_{\bar{k}}, \mathbf{Q}_{\ell}\left(\frac{n}{2}\right)\right)$ defines an orthogonal representation of the absolute Galois group $\Gamma_{k}=\operatorname{Gal}\left(k_{s} / k\right)$. The determinant

$$
\operatorname{det} V: \Gamma_{k} \rightarrow\{ \pm 1\} \subset \mathbf{Q}_{\ell}^{\times}
$$

is independent of the choice of $\ell$ (see Corollary 3.3).

Assume that $X$ is a smooth hypersurface of degree $d$ in a projective space of dimension $n+1$, and let $f$ be a homogeneous polynomial defining it. Let $\operatorname{disc}_{d}(f)$ be the divided discriminant of $f$ (see Section 2). Assume further that the characteristic of $k$ is not 2. We shall prove below (Theorem 3.5):

Theorem. The quadratic character $\operatorname{det} V$ is defined by the square root of $\varepsilon(n, d)$. $\operatorname{disc}_{d}(f)$, where $\varepsilon(n, d)$ is $(-1)^{\frac{d-1}{2}}$ if $d$ is odd and is $(-1)^{\frac{d}{2} \cdot \frac{n+2}{2}}$ if $d$ is even.

[In other words, the kernel of det $V: \Gamma_{k} \rightarrow\{ \pm 1\}$ is the subgroup of $\Gamma_{k}$ corresponding to the field extension $k\left(\sqrt{\varepsilon(n, d) \cdot \operatorname{disc}_{d}(f)}\right) / k$.]

The proof ${ }^{1}$ is in two parts. One shows (cf. Section 3) by a standard argument on universal families that the theorem is true up to a sign depending only on $d$ and $n$. One then concludes that this sign is equal to $\varepsilon(n, d)$, using a topological computation given in Section 1.

\section{Determinant of the complex conjugation}

We compute the determinant of the action of the complex conjugation on the cohomology of a proper smooth variety of even dimension over $\mathbf{R}$.

\footnotetext{
Received by the editors October 8, 2011.

${ }^{1}$ For $n=2$, this had already been proved by the late Torsten Ekedahl, in an unpublished 2-page manuscript dated February 1993. He was then answering a question raised by Jean-Pierre Serre in a Collège de France course on Galois representations and motives. Ekedahl's method was similar, but not identical, to the one in this article. The author was happy to be able to discuss these questions with him in november 2011, a few days before his unexpected death.
} 
1.1. We put $\Gamma_{\mathbf{R}}=\operatorname{Gal}(\mathbf{C} / \mathbf{R})=\{1, c\}$. We identify the Grothendieck group $K_{0}\left(\mathbf{Q}\left[\Gamma_{\mathbf{R}}\right]\right)$ of representations of $\Gamma_{\mathbf{R}}$ over $\mathbf{Q}$ with the group $\{(a, b) \in \mathbf{Z} \times \mathbf{Z} \mid a \equiv b(\bmod 2)\}$ by the isomorphism sending the class $[\rho]$ of a representation $\rho$ to the pair $(\operatorname{Tr} \rho(1), \operatorname{Tr} \rho(c))$. For a representation $V$ of $\Gamma_{\mathbf{R}}$ and an integer $m$, let $V(m)$ denote the twist of $V$ by the $m$ th power of the non-trivial character $\Gamma_{\mathbf{R}} \rightarrow\{ \pm 1\}$. The automorphism of $K_{0}\left(\mathbf{Q}\left[\Gamma_{\mathbf{R}}\right]\right)$ sending the class $[V]$ to $[V(1)]$ is given by $(a, b) \mapsto(a,-b)$. The map $\operatorname{det}: K_{0}\left(\mathbf{Q}\left[\Gamma_{\mathbf{R}}\right]\right) \rightarrow\{ \pm 1\}$ sending the class $[\rho]$ to $\operatorname{det} \rho(c)$ is given by $(a, b) \mapsto(-1)^{\frac{a-b}{2}}$.

Proposition 1.2. Let $X$ be a separated scheme of finite type of dimension $n$ over $\mathbf{R}$ and let $e_{\mathbf{C}}$ and $e_{\mathbf{R}}$ denote the Euler-Poincaré characteristics with compact support of the topological spaces $X(\mathbf{C})$ and $X(\mathbf{R})$ respectively. Then, the alternating sum

$$
\left[H_{c}^{*}(X(\mathbf{C}), \mathbf{Q})\right]=\sum_{q=0}^{2 n}(-1)^{q}\left[H_{c}^{q}(X(\mathbf{C}), \mathbf{Q})\right],
$$

viewed as an element of $K_{0}\left(\mathbf{Q}\left[\Gamma_{\mathbf{R}}\right]\right) \subset \mathbf{Z} \times \mathbf{Z}$, is equal to $\left(e_{\mathbf{C}}, e_{\mathbf{R}}\right)$.

Proof. It suffices to show that $e_{\mathbf{R}}=\operatorname{Tr}\left(c: H_{c}^{*}(X(\mathbf{C}), \mathbf{Q})\right)$. By the long exact sequence

$$
\cdots \rightarrow H_{c}^{*}(X(\mathbf{C})-X(\mathbf{R}), \mathbf{Q}) \rightarrow H_{c}^{*}(X(\mathbf{C}), \mathbf{Q}) \rightarrow H_{c}^{*}(X(\mathbf{R}), \mathbf{Q}) \rightarrow \cdots
$$

we have

$$
\operatorname{Tr}\left(c: H_{c}^{*}(X(\mathbf{C}), \mathbf{Q})\right)=\operatorname{Tr}\left(c: H_{c}^{*}(X(\mathbf{R}), \mathbf{Q})\right)+\operatorname{Tr}\left(c: H_{c}^{*}(X(\mathbf{C})-X(\mathbf{R}), \mathbf{Q})\right) .
$$

Since $X(\mathbf{R})$ consists of the $c$-fixed points, we have

$$
\operatorname{Tr}\left(c: H_{c}^{*}(X(\mathbf{R}), \mathbf{Q})\right)=e_{\mathbf{R}} .
$$

We now show that $\operatorname{Tr}\left(c: H_{c}^{*}(X(\mathbf{C})-X(\mathbf{R}), \mathbf{Q})\right)=0$. By a standard dévissage, we can reduce the question to the case where $X$ is affine, and then to the case where it is projective. Hence, we may assume that $X$ is a closed subscheme of a projective space $\mathbf{P}_{\mathbf{R}}^{m}$ over $\mathbf{R}$. The space $\mathbf{P}^{m}(\mathbf{C})$ can be embedded as a compact algebraic subset ([3, Definition 2.1.1]) in the space of $m+1$ by $m+1$ Hermitian matrices by the immersion sending the vector $z=\left(z_{0}, \ldots, z_{m}\right)$ to the matrix $\frac{{ }^{t} \bar{z} \cdot z}{\bar{z} \cdot{ }^{\cdot} z}$.

By [3, Proposition 2.2.7], this implies that the quotient $X(\mathbf{C}) / \Gamma_{\mathbf{R}}$ is homeomorphic to a compact semi-algebraic subset ([3, Definition 2.1.3]) of $\mathbf{R}^{(m+1)^{2}}$. Hence by [3, Théorème 9.2.1], there exists a triangulation of $X(\mathbf{C}) / \Gamma_{\mathbf{R}}$ such that the image of $X(\mathbf{R})$ is a union of simplices. For each simplex $\Delta$ in the triangulation, the inverse image of $\Delta-(\Delta \cap \operatorname{Image}(X(\mathbf{R})))$ in $X(\mathbf{C})$ consists of its 2 copies switched by $c$. Hence, the equality $\operatorname{Tr}\left(c: H_{c}^{*}(X(\mathbf{C})-X(\mathbf{R}), \mathbf{Q})\right)=0$ follows.

Corollary 1.3. Assume that $X$ is proper and smooth of even dimension $n$.

1. If we put $N=\frac{1}{2}\left(e_{\mathbf{C}}-(-1)^{\frac{n}{2}} e_{\mathbf{R}}\right)$, we have

$$
\operatorname{det}\left(c: H^{n}\left(X(\mathbf{C}), \mathbf{Q}\left(\frac{n}{2}\right)\right)\right)=(-1)^{N} .
$$

2. Let $X_{d}$ be the Fermat hypersurface in $\mathbf{P}_{\mathbf{R}}^{n+1}$ of degreed defined by the equation

$$
T_{0}^{d}+\cdots+T_{n+1}^{d}=0 .
$$


Then:

$$
\operatorname{det}\left(c: H^{n}\left(X_{d}(\mathbf{C}), \mathbf{Q}\left(\frac{n}{2}\right)\right)\right)= \begin{cases}(-1)^{\frac{d-1}{2}} & \text { if } d \text { is odd, } \\ (-1)^{\frac{d}{2} \frac{n+2}{2}} & \text { if } d \text { is even. }\end{cases}
$$

Proof. 1. Let $m=n / 2$. Recall that $\mathbf{Q}(m)$ is the $m$ th twist of $\mathbf{Q}$, as defined in 1.1; this means that the complex conjugation acts on it by $(-1)^{m}$.

We have $\operatorname{det}\left(c: H^{n}(X(\mathbf{C}), \mathbf{Q}(m))\right)=\operatorname{det}\left(c:\left[H^{*}(X(\mathbf{C}), \mathbf{Q}(m))\right]\right)$ by Poincaré duality. By Proposition 1.2, the class $\left[H^{*}(X(\mathbf{C}), \mathbf{Q}(m))\right]$ in $K_{0}\left(\mathbf{Q}\left[\Gamma_{\mathbf{R}}\right]\right)$ is $\left(e_{\mathbf{C}},(-1)^{m} e_{\mathbf{R}}\right)$ and we obtain $\operatorname{det}\left(c:\left[H^{*}(X(\mathbf{C}), \mathbf{Q}(m))\right]\right)=(-1)^{N}$.

2. If $d$ is odd, the morphism $\left(t_{0}, \ldots, t_{n+1}\right) \mapsto\left(t_{0}^{d}, \ldots, t_{n+1}^{d}\right)$ of $X_{d}$ into $X_{1}$ induces a homeomorphism $X_{d}(\mathbf{R}) \rightarrow X_{1}(\mathbf{R})$; since $X_{1}$ is isomorphic to $\mathbf{P}^{n}$, we have $e_{\mathbf{R}}=1$. If $d$ is even, then $X_{d}(\mathbf{R})=\varnothing$ and we have $e_{\mathbf{R}}=0$.

Let us now define a polynomial $\Phi(T) \in \mathbf{Z}[T]$ by

$$
\Phi(T)=\frac{1}{T} \cdot\left((1-T)^{n+2}-(1-(n+2) T)\right) .
$$

A standard computation using Chern classes shows that $e_{\mathbf{C}}=\Phi(d)$.

[For the convenience of the reader, we recall this computation. The exact sequences

$$
0 \rightarrow \Omega_{\mathbf{P}^{n+1}}^{1} \rightarrow \mathcal{O}_{\mathbf{P}^{n+1}}(-1)^{\oplus n+2} \rightarrow \mathcal{O}_{\mathbf{P}^{n+1}} \rightarrow 0
$$

and

$$
0 \rightarrow \mathcal{O}_{X_{d}}(-d) \rightarrow \Omega_{\mathbf{P}^{n+1}}^{1} \otimes \mathcal{O}_{X_{d}} \rightarrow \Omega_{X_{d}}^{1} \rightarrow 0
$$

imply that the total Chern class $c\left(\Omega_{X_{d}}^{1}\right)$ is equal to

$$
c(\mathcal{O}(-1))^{n+2} \cdot c(\mathcal{O}(-d))^{-1} \cdot\left[X_{d}\right]=(1-h)^{n+2} \cdot(1-d h)^{-1} \cdot d h,
$$

where $h=c_{1}(\mathcal{O}(1))$ denotes the class of a hyperplane. The coefficient of $h^{n+1}$ in $(-1)^{n} c\left(\Omega_{X_{d}}^{1}\right)$ is $(-1)^{n} \sum_{i=0}^{n}\left(\begin{array}{c}n+2 \\ i\end{array}\right)(-1)^{i} d^{n-i+1}=\sum_{j=2}^{n+2}\left(\begin{array}{c}n+2 \\ j\end{array}\right)(-1)^{j} d^{j-1}$ and is equal to $\frac{1}{d} \cdot\left((1-d)^{n+2}-(1-(n+2) d)\right)=\Phi(d)$.]

We have

$$
\Phi(d) \equiv \Phi(a)+(d-a) \Phi^{\prime}(a) \quad(\bmod 4) \quad \text { if } d \equiv a \quad(\bmod 2) .
$$

Since $\Phi(1)=n+1$ and $\Phi(1)+\Phi^{\prime}(1)=n+2$, we have $\Phi^{\prime}(1)=1$. Hence, if $d$ is odd, we get $\Phi(d) \equiv n+1+d-1 \equiv(-1)^{n / 2}+d-1(\bmod 4)$, and

$$
2 N \equiv(-1)^{n / 2}+d-1-(-1)^{n / 2} \equiv d-1 \quad(\bmod 4) .
$$

If $d$ is even, since $\Phi(0)=0$ and $\Phi^{\prime}(0)=\left(\begin{array}{c}n+2 \\ 2\end{array}\right) \equiv 1+\frac{n}{2}(\bmod 2)$, we have

$$
2 N=\Phi(d) \equiv d\left(1+\frac{n}{2}\right) \quad(\bmod 4)
$$

Hence, assertion 2 follows from assertion 1.

\section{Discriminant}

The literature contains several non-equivalent definitions of "the" discriminant of a homogeneous polynomial. For instance, the discriminant of $x^{2}+y^{2}$ is sometimes defined as 1 , sometimes as 4 and sometimes as -4 . In what follows, we shall put indices to the symbol "disc" in order to clarify the conventions we use.

We start with the most standard definition (see e.g., [10, Chap. 13]), which is satisfactory over $\mathbf{C}$, but which is not so over an arbitrary field. 


\subsection{The discriminant, defined as the resultant of the partial derivatives.} We fix integers $n \geqslant 0$ and $d>1$. We consider the polynomial $\operatorname{ring} \mathbf{Z}\left[T_{0}, \ldots, T_{n+1}\right]$ and the free $\mathbf{Z}$-module $E=\bigoplus_{i=0}^{n+1} \mathbf{Z} \cdot T_{i}$. The $d$ th symmetric power $S^{d} E$ is identified with the free $\mathbf{Z}$-module of finite rank consisting of homogeneous polynomials of degree $d$ in $\mathbf{Z}\left[T_{0}, \ldots, T_{n+1}\right]$. If $I=\left(i_{0}, \ldots, i_{n+1}\right) \in \mathbf{N}^{n+2}$ is a multi-index, we put

$$
T^{I}=T_{0}^{i_{0}} \cdots T_{n+1}^{i_{n+1}} \in \mathbf{Z}\left[T_{0}, \ldots, T_{n+1}\right] \text { and }|I|=i_{0}+\cdots+i_{n+1} .
$$

The monomials $T^{I}$ of degree $|I|=d$ form a basis of $S^{d} E$. Let $\left(C_{I}\right)_{|I|=d}$ be the dual basis of $\left(S^{d} E\right)^{\vee}$ and define the universal polynomial $F=\sum_{|I|=d} C_{I} T^{I}$.

We consider the resultant ${ }^{2}$

$$
\operatorname{res}\left(D_{0} F, \ldots, D_{n+1} F\right)
$$

of its partial derivatives $D_{0} F, \ldots, D_{n+1} F$. It is a homogeneous polynomial of degree $m=(n+2)(d-1)^{n+1}$ in $\left(C_{I}\right)_{|I|=d}$ with integral coefficients. If we put

$$
a(n, d)=\frac{(d-1)^{n+2}-(-1)^{n+2}}{d}
$$

the greatest common divisor of the coefficients is $d^{a(n, d)}$ by $\left[6, \mathrm{n}^{\circ} 5\right.$ Lemme 11] and [10, Chap. 13.1.D Proposition 1.7].

Definition 2.2. We call $\operatorname{res}\left(D_{0} F, \ldots, D_{n+1} F\right)$ the resultant-discriminant of $F$ and we denote it by $\operatorname{disc}_{r}(F)$. We call

$$
\operatorname{disc}_{d}(F)=\frac{1}{d^{a(n, d)}} \operatorname{disc}_{r}(F)
$$

the divided discriminant of $F$.

The divided discriminant $\operatorname{disc}_{d}(F)$ is known to be geometrically irreducible in characteristic $0 \mathrm{cf}$. [6, $\mathrm{n}^{\mathrm{O}} 6$ Proposition 14] and [10, Chap. 13.1.D]. It is proved purely algebraically in $[6$, loc. cit.]. The proof in [10, loc. cit.] is geometric and is generalized to positive characteristic in Lemma 2.12 below, except the exceptional case in characteristic 2. See also the remark at the end of this section.

By specialization, the definition of discriminants gives a meaning to $\operatorname{disc}_{r}(f)$ and $\operatorname{disc}_{d}(f)$ for every homogeneous polynomial $f$ in $n+2$ variables over a commutative ring $R$. The divided discriminant satisfies the following smoothness criterion due to Demazure:

Proposition 2.3 ([6, $\mathrm{n}^{\circ} 5$ Proposition 12]). Let $f$ be a homogeneous polynomial of degree $d$ in $n+2$ variables over a commutative ring $R$. Then, the divided discriminant $\operatorname{disc}_{d}(f)$ is invertible in $R$ if and only if the corresponding hypersurface is a smooth divisor of the projective space $\mathbf{P}_{R}^{n+1}=\operatorname{Proj} R\left[T_{0}, \ldots, T_{n+1}\right]$ over $R$.

This smoothness criterion would not work with the resultant-discriminant when $d$ is not invertible in $R$.

The transformation properties of $\operatorname{disc}_{d}$ are the same as those of $\operatorname{disc}_{r}$, namely (cf. [6, $\mathrm{n}^{\mathrm{o}} 5$ Proposition 11] and [10]):

$$
\operatorname{disc}_{d}(\lambda f)=\lambda^{(n+2)(d-1)^{n+1}} \operatorname{disc}_{d}(f)
$$

\footnotetext{
${ }^{2}$ For the definition of the resultant of $m$ homogeneous polynomials in $m$ indeterminates, see e.g., [10, Chap.13, Section 1.A] and [6, n⿳0 4 Définition 3].
} 
and

$$
\operatorname{disc}_{d}\left(f_{A}\right)=\operatorname{det}(A)^{d(d-1)^{n+1}} \operatorname{disc}_{d}(f),
$$

where $\lambda$ is any element of $R, A$ is any $(n+2) \times(n+2)$-matrix with coefficients in $R$, and $f_{A}(x)=f(A x)$. The formula about $f_{A}$, applied to a permutation, shows that $\operatorname{disc}_{d}(f)$ does not depend on the indexing of the coordinates.

2.4. The universal family and the discriminant. We define the universal family of hypersurfaces. We put $\mathbf{P}_{\mathbf{Z}}^{n+1}=\mathbf{P}(E)=\operatorname{Proj} \mathbf{Z}\left[T_{0}, \ldots, T_{n+1}\right]$. Let $\mathbf{P}_{n, d}=$ $\mathbf{P}\left(\left(S^{d} E\right)^{\vee}\right)$ be the projective space defined by the dual $\left(S^{d} E\right)^{\vee}=\operatorname{Hom}\left(S^{d} E, \mathbf{Z}\right)$; it is the moduli space of hypersurfaces of degree $d$ in $\mathbf{P}^{n+1}$; we shall usually write it $\mathbf{P}$ instead of $\mathbf{P}_{n, d}$. The universal hypersurface

$$
X \subset \mathbf{P}_{\mathrm{Z}}^{n+1} \times \mathbf{P}
$$

is then defined by the universal polynomial $F=\sum_{|I|=d} C_{I} T^{I}$.

Let $\Delta$ be the closed subscheme of $X$ defined by the vanishing of the partial derivatives $D_{0} F, \ldots, D_{n+1} F$ of the universal polynomial $F$. By the Jacobian criterion, $X-\Delta$ is the maximum open subscheme of $X$ on which the canonical morphism $X \rightarrow \mathbf{P}$ is smooth.

For an integer $m \geq 0$, we identify the $\mathbf{Z}$-module $\Gamma(\mathbf{P}, \mathcal{O}(m))$ with the symmetric power $S^{m}\left(\left(S^{d} E\right)^{\vee}\right)$ consisting of homogeneous polynomials in $\left(C_{I}\right)_{|I|=d}$ of degree $m$. The closed subscheme $D$ of $\mathbf{P}=\mathbf{P}_{n, d}$ defined by the divided discriminant $\operatorname{disc}_{d}(F)$ is reduced and is an effective Cartier divisor flat over $\mathbf{Z}$. For $m=(n+2)(d-1)^{n+1}$, the divided discriminant $\operatorname{disc}_{d}(F)$ is a basis of the free $\mathbf{Z}$-module $L=\Gamma(\mathbf{P}, \mathcal{O}(m)(-D))$ of rank 1.

The following is a geometric interpretation of the smoothness criterion Proposition 2.3.

Lemma 2.5. The underlying set of the reduced closed subscheme $D$ of $\mathbf{P}$ equals the image of $\Delta$ by the projection map $\mathbf{P}_{\mathbf{Z}}^{n+1} \times \mathbf{P} \rightarrow \mathbf{P}$.

The equality of underlying sets means that the scheme theoretic image of $\Delta \rightarrow \mathbf{P}$ equals the reduced closed subscheme $D$ of $\mathbf{P}$.

2.6. The discriminant and the dual variety. We recall the formalism of the dual variety $[13,3.1]$ and the relation with the discriminant. The rest of this section is not used in the proof of Theorem 3.5 except the description in Lemma 2.10 at the generic point of the divisor $D_{k}$ recalled from SGA 7.

In this subsection, we fix an algebraically closed field $k$ and let the suffix $k$ denote the base change to $k$ over $\mathbf{Z}$. The indeterminates $T_{0}, \ldots, T_{n+1}$ define a homogeneous coordinate of the projective space $\mathbf{P}_{k}^{n+1}=\mathbf{P}\left(E_{k}\right)$. We put $r=\operatorname{dim}\left(S^{d} E_{k}\right)-1$ and $\mathbf{P}^{r}=\mathbf{P}\left(S^{d} E_{k}\right)$. The projective space $\mathbf{P}_{k}=\mathbf{P}\left(\left(S^{d} E_{k}\right)^{\vee}\right)$ is the dual $\check{\mathbf{P}}^{r}$ of $\mathbf{P}^{r}$ parametrizing hyperplanes in $\mathbf{P}^{r}$. The monomials $\left(T^{I}\right)_{|I|=d}$ form a homogeneous coordinate of $\mathbf{P}^{r}$ and the universal coefficients $\left(C_{I}\right)_{|I|=d}$ form a homogeneous coordinate of $\check{\mathbf{P}}^{r}=\mathbf{P}_{k}$.

The $d$-th symmetric power $S^{d} E_{k}$ is naturally identified with the space of global sections $\Gamma\left(\mathbf{P}_{k}^{n+1}, \mathcal{O}(d)\right)$ of the very ample invertible sheaf $\mathcal{O}(d)$ on $\mathbf{P}_{k}^{n+1}$. The closed immersion

$$
v_{d}: \mathbf{P}_{k}^{n+1}=\mathbf{P}\left(E_{k}\right) \rightarrow \mathbf{P}^{r}=\mathbf{P}\left(S^{d} E_{k}\right)
$$


defined by $\mathcal{O}(d)$ on $\mathbf{P}_{k}^{n+1}$ is called the $d$ th Veronese embedding. We consider $\mathbf{P}_{k}^{n+1}$ as a closed subscheme of $\mathbf{P}^{r}$ by $v_{d}$.

The conormal sheaf $\mathcal{N}$ of $\mathbf{P}_{k}^{n+1}$ in $\mathbf{P}^{r}$ is defined as $\mathcal{I} / \mathcal{I}^{2}$ where $\mathcal{I} \subset \mathcal{O}_{\mathbf{P}^{r}}$ denotes the ideal sheaf defining the closed subscheme $\mathbf{P}_{k}^{n+1} \subset \mathbf{P}^{r}$. It is a locally free $\mathcal{O}_{\mathbf{P}_{k}^{n+1-}}$ module of rank $r-(n+1)$. Let $\mathbb{P}(\mathcal{N})=\operatorname{Proj} S^{\bullet} \mathcal{N}^{\vee}$ denote the associated covariant projective space bundle over $\mathbf{P}_{k}^{n+1}$ [here, to be consistent with [13, 3.1], we use the font $\mathbb{P}$ to denote the covariant projective space bundle associated to a locally free sheaf, parametrizing the lines].

We view the projective space bundle $\mathbb{P}(\mathcal{N})$ over $\mathbf{P}_{k}^{n+1}$ as a closed subscheme of $\mathbf{P}_{k}^{n+1} \times \mathbf{P}_{k}$ as in $[13,(3.1 .1)]$. We recall the definition of the embedding

$$
\mathbb{P}(\mathcal{N}) \rightarrow \mathbf{P}_{k}^{n+1} \times \mathbf{P}_{k}
$$

The projective space $\mathbf{P}_{k}=\mathbf{P}\left(\left(S^{d} E_{k}\right)^{\vee}\right)$ is the covariant projective space $\mathbb{P}\left(S^{d} E_{k}\right)$ associated to the vector space $S^{d} E_{k}$. Since the twist by an invertible sheaf does not change the associated projective space bundle, the product $\mathbf{P}_{k}^{n+1} \times \mathbf{P}_{k}$ is identified with the covariant projective space bundle $\mathbb{P}\left(S^{d} E_{k} \otimes v_{d}^{*} \mathcal{O}_{\mathbf{P}^{r}}(-1)\right)$ associated to a locally

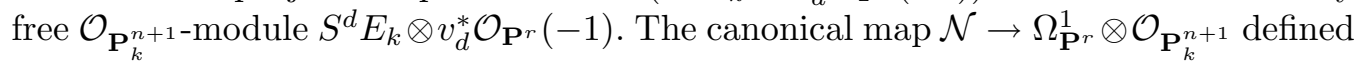
by the closed immersion $\mathbf{P}_{k}^{n+1} \rightarrow \mathbf{P}^{r}$ and the canonical map $\Omega_{\mathbf{P}^{r}}^{1} \rightarrow S^{d} E_{k} \otimes \mathcal{O}_{\mathbf{P}^{r}(-1)}$ for the projective space $\mathbf{P}^{r}=\mathbf{P}\left(S^{d} E_{k}\right)$ are locally splitting injections. Hence the composition $\mathcal{N} \rightarrow \Omega_{\mathbf{P}^{r}}^{1} \otimes \mathcal{O}_{\mathbf{P}_{k}^{n+1}} \rightarrow S^{d} E_{k} \otimes v_{d}^{*} \mathcal{O}_{\mathbf{P}^{r}}(-1)$ defines the desired closed immersion $\mathbb{P}(\mathcal{N}) \rightarrow \mathbf{P}_{k}^{n+1} \times \mathbf{P}_{k}$ by the covariant functoriality.

The map $\varphi: \mathbb{P}(\mathcal{N}) \rightarrow \check{\mathbf{P}}^{r}[13,(3.1 .2)]$ is defined as the composition

$$
\mathbb{P}(\mathcal{N}) \rightarrow \mathbf{P}_{k}^{n+1} \times \mathbf{P}_{k} \rightarrow \mathbf{P}_{k}
$$

of the immersion (2.4) and the projection. The dual variety of $\mathbf{P}_{k}^{n+1} \subset \mathbf{P}_{k}^{r}$ is the closed subset defined as the image of $\varphi[13,(3.1 .3)]$.

Lemma 2.7. Let $k$ be an algebraically closed field.

1. We have an equality $\mathbb{P}(\mathcal{N})=\Delta_{k}$ of the underlying sets of closed subschemes of $\mathbf{P}_{k}^{n+1} \times \mathbf{P}_{k}$.

2. The dual variety of $\mathbf{P}_{k}^{n+1}$ in $\mathbf{P}_{k}^{r}$ defined as the image of the map $\varphi: \mathbb{P}(\mathcal{N}) \rightarrow \mathbf{P}_{k}$ (2.5) equals the underlying set of $D_{k}$.

The equality $\mathbb{P}(\mathcal{N})=\Delta_{k}$ of the underlying sets will imply the isomorphism of projective space bundles as a consequence of Proposition 2.8 below.

Proof. 1. A $k$-rational point of $\mathbf{P}(k)$ corresponds to a hyperplane $H$ in $\mathbf{P}^{r}$ defined over $k$ since $\mathbf{P}_{k}$ is the dual of $\mathbf{P}^{r}$. The intersection of $H$ with $\mathbf{P}_{k}^{n+1}$ embedded in $\mathbf{P}^{r}$ by the $d$ th Veronese embedding $v_{d}: \mathbf{P}_{k}^{n+1} \rightarrow \mathbf{P}^{r}$ is a hypersurface of degree $d$ and is the geometric fiber of the universal family $X \rightarrow \mathbf{P}$ at the geometric point $[H] \in \mathbf{P}(k)$. Consequently, the set of $k$-valued points $X(k) \subset \mathbf{P}^{n+1}(k) \times \mathbf{P}(k)$ consists of the pairs $(x, H) \in \mathbf{P}^{n+1}(k) \times \mathbf{P}(k)$, where $H$ is a hyperplane of $\mathbf{P}^{r}$ containing $x \in \mathbf{P}^{n+1}(k) \subset \mathbf{P}^{r}(k)$.

By [13, (3.1.1)], the set of $k$-valued points $\mathbb{P}(\mathcal{N})(k) \subset \mathbf{P}^{n+1}(k) \times \mathbf{P}(k)$ consists of the pairs $(x, H) \in X(k)$ such that $H$ is tangent to $\mathbf{P}_{k}^{n+1}$ at $x$. In other words, it consists of the points of $X(k) \subset \mathbf{P}^{n+1}(k) \times \mathbf{P}(k)$ where the geometric fiber $X_{k} \rightarrow \mathbf{P}_{k}$ 
of the canonical map of the universal family $X \rightarrow \mathbf{P}$ is not smooth. Thus the assertion follows by the Jacobian criterion.

2. Since the dual variety is defined as the image $\varphi(\mathbb{P}(\mathcal{N}))$, it follows from assertion 1 and the smoothness criterion Proposition 2.3.

Proposition 2.8. $\Delta$ is a projective space bundle over $\mathbf{P}_{\mathbf{Z}}^{n+1}$.

By Lemma 2.7.1, we already know that the reduced part of each geometric fiber $\Delta_{k}$ is a projective space bundle. We verify that the global equations defining $\Delta$ reduce Proposition 2.8 to this property.

Proof. The closed subscheme $\Delta$ of $\mathbf{P}_{\mathbf{Z}}^{n+1} \times \mathbf{P}$ is defined, on the inverse image of the open subscheme $D\left(T_{i}\right)$ of $\mathbf{P}_{\mathbf{Z}}^{n+1}$, by the vanishing of the $n+2$ linear forms $F, D_{0} F, \ldots, D_{i-1} F, D_{i+1} F, \ldots, D_{n+1} F$ in $\left(C_{I}\right)_{|I|=d}$ by the equality

$$
d \cdot F=T_{0} \cdot D_{0} F+\cdots+T_{n+1} \cdot D_{n+1} F .
$$

Hence, it suffices to show that for every geometric point of each $D\left(T_{i}\right)$, these forms are linearly independent. Or equivalently, it suffices to show that for every geometric point Spec $k \rightarrow \mathbf{P}_{\mathbf{Z}}^{n+1}$, the geometric fiber of $\Delta_{k} \rightarrow \mathbf{P}_{k}^{n+1}$ is a linear subspace of $\mathbf{P}_{k}$ of codimension $n+2$.

Let $k$ be an algebraically closed field and take the notation in 2.6. By Lemma 2.7.1, we have an equality $\Delta_{k}=\mathbb{P}(\mathcal{N})$ of the underlying sets. By the definition of the closed immersion $\mathbb{P}(\mathcal{N}) \rightarrow \mathbf{P}_{k}^{n+1} \times \mathbf{P}_{k}(2.4)$ recalled above, $\mathbb{P}(\mathcal{N})$ is a linear subbundle of the projective space bundle $\mathbf{P}_{k}^{n+1} \times \mathbf{P}_{k}$ over $\mathbf{P}_{k}^{n+1}$. Hence the fiber of $\Delta_{k}=\mathbb{P}(\mathcal{N}) \rightarrow \mathbf{P}_{k}^{n+1}$ is a linear subspace of $\mathbf{P}_{k}$ of codimension $\operatorname{dim}\left(\mathbf{P}_{k}^{n+1} \times \mathbf{P}_{k}\right)-\operatorname{dim} \mathbb{P}(\mathcal{N})$.

Since rank $\mathcal{N}=\operatorname{dim} \mathbf{P}_{k}-\operatorname{dim} \mathbf{P}_{k}^{n+1}$, the codimension $\operatorname{dim}\left(\mathbf{P}_{k}^{n+1} \times \mathbf{P}_{k}\right)-\operatorname{dim} \mathbb{P}(\mathcal{N})$ is equal to $\left(\operatorname{dim} \mathbf{P}_{k}+\operatorname{dim} \mathbf{P}_{k}^{n+1}\right)-\left(\operatorname{dim} \mathbf{P}_{k}-\operatorname{dim} \mathbf{P}_{k}^{n+1}-1+\operatorname{dim} \mathbf{P}_{k}^{n+1}\right)=n+2$. Thus, the assertion follows.

2.9. The irreducibility of the discriminant modulo $p$. Let $\pi: X \rightarrow \mathbf{P}$ denote the canonical morphism of the universal family of hypersurfaces. By $[5$, Corollaire 1.3.4] there exists an open subset $W$ of $\Delta$ consisting of the points $w$ such that $w$ is an ordinary quadratic singularity in the fiber $X_{\pi(w)}$.

Lemma 2.10. 1. For every algebraically closed field $k$, the geometric fiber $W_{k}$ is dense in $\mathbb{P}(\mathcal{N})=\Delta_{k}$.

2. Assume that $n$ is odd or char $k \neq 2$. Let $W_{k}^{\prime}$ be the subset of $W_{k} \subset \mathbb{P}(\mathcal{N})$ consisting of the images of geometric points $w$ of $W_{k}$ that is a unique singular point in the geometric fiber $X_{\pi(w)}$.

Then, $W_{k}^{\prime}$ is the maximum open subscheme of $\mathbb{P}(\mathcal{N})$ where the restriction of the morphism $\varphi: \mathbb{P}(\mathcal{N}) \rightarrow \mathbf{P}_{k}$ is an immersion. Consequently, the canonical morphism $\mathbb{P}(\mathcal{N})=\Delta_{k} \rightarrow D_{k, \text { red }}$ to the maximum reduced subscheme of $D_{k}$ is birational.

Proof. 1. By $[13,(3.7 .1)]$, the open subscheme $W_{k}$ of $\mathbb{P}(\mathcal{N})$ is non-empty. Since $\mathbb{P}(\mathcal{N})$ is irreducible, it is dense.

2. By assertion 1 and [13, Proposition 3.3], the morphism $\varphi: \mathbb{P}(\mathcal{N}) \rightarrow \mathbf{P}_{k}$ (2.5) is generically unramified ("unramified" as defined in EGA IV (17.3.1) is the same as "net" in SGA 7: the diagonal map is an open immersion). Hence, the assertion follows from [13, Proposition 3.5]. 
We deduce the irreducibility of the reduction of the discriminant modulo $p$ from Proposition 2.8 using the following Lemma.

Lemma 2.11. Let $f: X \rightarrow Y$ be a proper morphism of Noetherian schemes. For a point $y$ of $Y$, let $f_{y}: X_{y} \rightarrow y$ denote the base change by the canonical map $y=$ Spec $\kappa(y) \rightarrow Y$ and define a subset $V$ of $Y$ by

$$
V=\left\{y \in Y \mid X_{y}=\emptyset \text { or } f_{y}: X_{y} \rightarrow y \text { is an isomorphism }\right\} .
$$

Then $V$ is the largest open subset of $Y$ such that $X \times_{Y} V \rightarrow V$ is a closed immersion.

Proof. The subset

$$
U=\left\{x \in X \mid x \text { is isolated in } f^{-1}(f(x))\right\}
$$

of $X$ is the largest open subset of $X$ such that the restriction $\left.f\right|_{U}: U \rightarrow Y$ is quasifinite by [11, Proposition (4.4.1)]. The complement $W=Y-f(X-U)$ is the largest open subset of $Y$ such that the base change $W \times_{Y} X \rightarrow W$ is finite by [11, Proposition (4.4.2)]. Since $V$ is a subset of $W$, by replacing $Y$ by $W$, we may assume $f$ is finite.

By Nakayama's lemma, the subset $V$ of $Y$ is the complement of the support of the coherent $\mathcal{O}_{Y}$-module $\operatorname{Coker}\left(\mathcal{O}_{Y} \rightarrow f_{*} \mathcal{O}_{X}\right)$. Hence $V$ is the largest open subscheme where the morphism $\mathcal{O}_{Y} \rightarrow f_{*} \mathcal{O}_{X}$ is a surjection.

Proposition 2.12. Unless $p=2$ and $n$ is even, the divided discriminant $\operatorname{disc}_{d}(F)$ modulo $p$ is a geometrically irreducible polynomial in the $C_{I}$. Consequently, the canonical morphism $\mathbb{P}(\mathcal{N})=\Delta_{\mathbf{F}_{p}} \rightarrow D_{\mathbf{F}_{p}}$ is birational.

Proof. Let $V$ be the largest open subset of $\mathbf{P}$ such that $\Delta \times_{\mathbf{P}} V \rightarrow V$ is a closed immersion. Since $\Delta$ is reduced by Proposition 2.8, the induced map $\Delta \times_{\mathbf{P}} V \rightarrow D \times_{\mathbf{P}} V$ is an closed immersion of reduced schemes and is an isomorphism by Lemmas 2.11 and 2.5 .

Since the construction of $V$ commutes with base change, Lemmas 2.10 .2 and 2.11 imply that the inverse image of $V_{\mathbf{F}_{p}}$ is dense in $\Delta_{\mathbf{F}_{p}}$ if $n$ is odd or $p \neq 2$. Since the geometric fiber $\Delta_{\overline{\mathbf{F}}_{p}}$ is reduced and irreducible by Proposition 2.8, the open subscheme $\left(D \times_{\mathbf{P}} V\right)_{\overline{\mathbf{F}}_{p}}$ of $D_{\overline{\mathbf{F}}_{p}}$ is reduced, irreducible and dense. Hence, the fiber $D_{\mathbf{F}_{p}}$ is a divisor of $\mathbf{P}_{\mathbf{F}_{p}}$ defined by a geometrically irreducible polynomial.

The last assertion follows from that in Lemma 2.10.2 and that $D_{\mathbf{F}_{p}}$ is reduced.

Proposition 2.12 is proved in [6, $\mathrm{n}^{\circ} 5$ Proposition 14] under the assumption $p \nmid$ $d(d-1)$. In the exceptional case $p=2$ and $n$ even, we show in Theorem 4.2 that the signed discriminant $\varepsilon(n, d) \operatorname{disc}_{d}(F)$ is congruent to a square modulo 4 where $\varepsilon(n, d)$ is defined as in Theorem stated in the introduction.

\section{Determinant}

In this section, we assume that $n$ is even. 
3.1. Let $S$ be a normal integral scheme of finite type over $\mathbf{Z}$ and $f: X \rightarrow S$ be a proper smooth morphism of relative dimension $n$. For a prime number $\ell$ invertible in the function field of $S$, the cup-product defines a non-degenerate symmetric bilinear

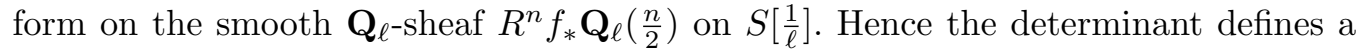
character $\pi_{1}\left(S\left[\frac{1}{\ell}\right]\right)^{a b} \rightarrow\{ \pm 1\} \subset \mathbf{Q}_{\ell}^{\times}$of the fundamental group, which we denote by $\left[\operatorname{det} H_{\ell}^{n}(X)\right]$.

Lemma 3.2. There exists a unique character $\left[\operatorname{det} H^{n}(X)\right]: \pi_{1}(S)^{a b} \rightarrow\{ \pm 1\}$ such that, for every prime number $\ell$ invertible in the function field of $S$, the composition with the map $\pi_{1}\left(S\left[\frac{1}{\ell}\right]\right)^{a b} \rightarrow \pi_{1}(S)^{a b}$ induced by the open immersion $S\left[\frac{1}{\ell}\right] \rightarrow S$ gives $\left[\operatorname{det} H_{\ell}^{n}(X)\right]$.

Proof. First, we show the case where $S=$ Spec $k$ for a finite field $k=\mathbf{F}_{q}$. Then, for a prime $\ell$ different form the characteristic of $k$, Deligne's theorem on $\ell$-adic representations tells us that the polynomial $\operatorname{det}\left(1-F r_{q} t: H^{n}\left(X_{\overline{\mathbf{F}}_{q}}, \mathbf{Q}_{\ell}\right)\right)$ is in $\mathbf{Z}[t]$ and is independent of $\ell$. Hence, the multiplicity $m$ of the eigenvalue of $-q^{n / 2}$ of $F r_{q}$ acting on $H^{n}\left(X_{\overline{\mathbf{F}}_{q}}, \mathbf{Q}_{\ell}\right)$ and the determinant $\operatorname{det}\left(F r_{q}: H^{n}\left(X_{\overline{\mathbf{F}}_{q}}, \mathbf{Q}_{\ell}\left(\frac{n}{2}\right)\right)\right)=(-1)^{m}$ are independent of $\ell$. Since the geometric Frobenius $F r_{q}$ generates a dense subgroup of $\Gamma_{k}$, the assertion follows in this case.

We prove the general case. For two different primes $\ell$ and $\ell^{\prime}$ and for every closed point $x$ of $S\left[\frac{1}{\ell \ell^{\prime}}\right]$, the case proved above gives us a commutative diagram

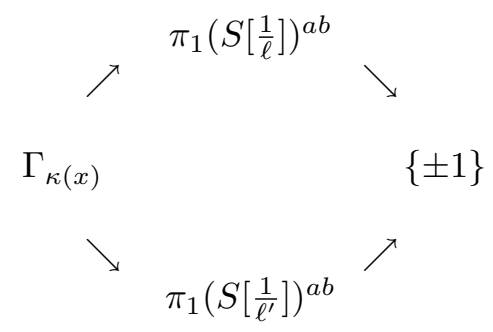

where the left slant arrows are induced by the closed immersion of $x$.

By the Chebotarev density theorem [16, Theorem 7], [17, Theorem 9.11] for a normal scheme of finite type over $\mathbf{Z}$, the images $\Gamma_{\kappa(x)} \rightarrow \pi_{1}\left(S\left[\frac{1}{\ell \ell^{\prime}}\right]\right)^{a b}$ where $x$ runs the closed points of $S\left[\frac{1}{\ell \ell^{\prime}}\right]$ generate a dense subgroup. Hence we obtain a commutative diagram

$$
\begin{aligned}
& \pi_{1}\left(S\left[\frac{1}{\ell}\right]\right)^{a b} \searrow \\
& \pi_{1}\left(S\left[\frac{1}{\ell \ell^{\prime}}\right]\right)^{a b} \quad\{ \pm 1\} . \\
& \pi_{1}\left(S\left[\frac{1}{\ell^{\prime}}\right]\right)^{a b}
\end{aligned}
$$

Glueing of coverings shows that $\pi_{1}(S)^{a b}$ is the push-out of the left half of (3.2). Thus, the characters $\left[\operatorname{det} H_{\ell}^{n}(X)\right]$ induce a unique character $\pi_{1}(S)^{a b} \rightarrow\{ \pm 1\}$, which is independent of $\ell$. 
Corollary 3.3. Let $X$ be a proper smooth scheme of even dimension $n$ over a field $k$. Then, for a prime number $\ell$ invertible in $k$, the character $\operatorname{det} H^{n}\left(X_{\bar{k}}, \mathbf{Q}_{\ell}\left(\frac{n}{2}\right)\right)$ of $\Gamma_{k}$ is independent of $\ell$.

Proof. We may assume that $k$ is finitely generated over a prime field. Hence, there exist a normal integral scheme $S$ of finite type over $\mathbf{Z}$ such that $k$ is the function field of $S$ and a proper smooth morphism $f: X_{S} \rightarrow S$ of relative dimension $n$ such that the generic fiber is $X$. Now the assertion follows form Lemma 3.2.

If $k$ contains an algebraic closure of $\mathbf{Q}$ as a subfield, it is reduced to the case where $k$ is finitely generated over $\mathbf{C}$ by a standard limit argument and the Lefschetz principle. In this case, one can deduce the independence of $\ell$ from the comparison theorem between Betti and $\ell$-adic cohomology.

3.4. Let $d>1$ be an integer and $\pi: X \subset \mathbf{P}_{\mathbf{Z}}^{n+1} \times \mathbf{P} \rightarrow \mathbf{P}=\mathbf{P}\left(\left(S^{d} E\right)^{\vee}\right)$ denote the universal hypersurface of degree $d$ defined by the universal polynomial $F$. The complement $U=\mathbf{P}-D$ of the divisor $D$ defined by the divided discriminant $\operatorname{disc}_{d}(F)$ is the maximum open subscheme of $\mathbf{P}$ over which $\pi: X \rightarrow \mathbf{P}$ is smooth.

Since $n$ is even, the degree $m=(n+2)(d-1)^{n+1}$ of the discriminant $\operatorname{disc}_{d}(F)$ is even. We consider the $\mu_{2}$-torsor on the fppf-site of $U$ consisting of bases of $\mathcal{O}\left(\frac{m}{2}\right)$ whose square is equal to the basis $\operatorname{disc}_{d}(F)$ of an invertible $\mathcal{O}_{U}$-module $\mathcal{O}_{U}(m)$. We denote by $\left[\operatorname{disc}_{d}(F)\right]$ the class of this torsor in $H_{\mathrm{f}}^{1}\left(U, \mu_{2}\right)$.

By applying Lemma 3.2 to the universal smooth hypersurface

$$
\pi_{U}: X_{U} \rightarrow U
$$

we define $\left[\operatorname{det} H^{n}(X)\right] \in H^{1}(U, \mathbf{Z} / 2 \mathbf{Z})$. Let now $k$ be a field and let $f \in S^{d} E \otimes$ $k$ be a homogeneous polynomial of degree $d$ defining a smooth hypersurface $Y$ in $\mathbf{P}_{k}^{n+1}$. Then, the pull-back in $H^{1}(k, \mathbf{Z} / 2 \mathbf{Z})=\operatorname{Hom}\left(\Gamma_{k}^{a b}, \mathbf{Z} / 2 \mathbf{Z}\right)$ of $\left[\operatorname{det} H^{n}(X)\right]$ by the $k$-valued point of $U$ corresponding to $f$ is given by the determinant of the orthogonal representation $H^{n}\left(Y_{\bar{k}}, \mathbf{Q}_{\ell}\left(\frac{n}{2}\right)\right)$ for a prime number $\ell$ invertible in $k$.

Theorem 3.5. Let $n \geqslant 0$ and $d>1$ be integers; assume that $n$ is even. Define the $\operatorname{sign} \varepsilon(n, d)= \pm 1$ by

$$
\varepsilon(n, d)= \begin{cases}(-1)^{\frac{d-1}{2}} & \text { if } d \text { is odd } \\ (-1)^{\frac{d}{2} \frac{n+2}{2}} & \text { if } d \text { is even }\end{cases}
$$

Then, we have

$$
\left[\operatorname{det} H^{n}(X)\right]=\left[\varepsilon(n, d) \cdot \operatorname{disc}_{d}(F)\right]
$$

in $H^{1}\left(U_{\mathbf{Z}\left[\frac{1}{2}\right]}, \mathbf{Z} / 2 \mathbf{Z}\right)$.

By a standard specialization argument, Theorem 3.5 implies the Theorem stated in the introduction.

Proof. The Kummer sequence defines an exact sequence

$$
0 \rightarrow \Gamma\left(U_{\frac{1}{2}}, \mathcal{O}\right)^{\times} /\left(\Gamma\left(U_{\frac{1}{2}}, \mathcal{O}\right)^{\times}\right)^{2} \rightarrow H^{1}\left(U_{\frac{1}{2}}, \mathbf{Z} / 2 \mathbf{Z}\right) \rightarrow \operatorname{Pic}\left(U_{\frac{1}{2}}\right)[2] \rightarrow 0
$$

where we have written $U_{\frac{1}{2}}$ instead of $U_{\mathbf{Z}\left[\frac{1}{2}\right]}$ for typographical reasons, and $\operatorname{Pic}\left(U_{\frac{1}{2}}\right)[2]$ denotes the subgroup of $\operatorname{Pic}\left(U_{\frac{1}{2}}\right)$ killed by 2 . 
We now compute $\Gamma\left(U_{\frac{1}{2}}, \mathcal{O}\right)^{\times}$and $\operatorname{Pic}\left(U_{\frac{1}{2}}\right)$. In the exact sequence,

$$
0 \rightarrow \Gamma\left(\mathbf{P}_{\mathbf{Z}\left[\frac{1}{2}\right]}, \mathcal{O}\right)^{\times} \rightarrow \Gamma\left(U_{\frac{1}{2}}, \mathcal{O}\right)^{\times} \rightarrow \mathbf{Z} \rightarrow \operatorname{Pic}\left(\mathbf{P}_{\mathbf{Z}\left[\frac{1}{2}\right]}\right) \rightarrow \operatorname{Pic}\left(U_{\frac{1}{2}}\right) \rightarrow 0
$$

the Picard group $\operatorname{Pic}\left(\mathbf{P}_{\mathbf{Z}\left[\frac{1}{2}\right]}\right)$ is canonically identified with $\mathbf{Z}$ by the generator $[\mathcal{O}(1)]$. Then, the map $\mathbf{Z} \rightarrow \operatorname{Pic}\left(\mathbf{P}_{\mathbf{Z}\left[\frac{1}{2}\right]}\right)$ is identified with the multiplication by $m=(n+$ $2)(d-1)^{n+1}$ since it sends 1 to the class of $\mathcal{O}(m)$. Thus, we have

$$
\Gamma\left(U_{\frac{1}{2}}, \mathcal{O}\right)^{\times}=\Gamma\left(\mathbf{P}_{\mathbf{Z}\left[\frac{1}{2}\right]}, \mathcal{O}\right)^{\times}=\mathbf{Z}\left[\frac{1}{2}\right]^{\times}=\langle-1,2\rangle .
$$

It also follows from (3.6) that the Picard group $\operatorname{Pic}\left(U_{\frac{1}{2}}\right)$ is cyclic of order $m$; since $m$ is even, this shows that $\operatorname{Pic}\left(U_{\frac{1}{2}}\right)[2]$ has order 2 .

Recall that the divisor $D$ defined by $\operatorname{disc}_{d} F$ is irreducible (see remark after Definition 2.2). Let $\bar{\xi}$ be a geometric generic point of $D$ and let $I_{\bar{\xi}}$ denote the absolute Galois group of the fraction field of the strict henselization $\mathcal{O}_{\mathbf{P}, \bar{\xi}}$. Since the profinite group $I_{\bar{\xi}}$ is isomorphic to $\widehat{\mathbf{Z}}$, the group $\operatorname{Hom}\left(I_{\bar{\xi}}, \mathbf{Z} / 2 \mathbf{Z}\right)$ is of order 2 .

We show that the images of $\left[\operatorname{det} H^{n}(X)\right]$ and $\left[\operatorname{disc}_{d}(F)\right]$ by the restriction map $H^{1}\left(U_{\frac{1}{2}}, \mathbf{Z} / 2 \mathbf{Z}\right) \rightarrow \operatorname{Hom}\left(I_{\bar{\xi}}, \mathbf{Z} / 2 \mathbf{Z}\right)$ are both the unique non-trivial element. For the latter $\left[\operatorname{disc}_{d}(F)\right]$, this follows from that $\operatorname{disc}_{d}(F)$ is a prime element of the irreducible divisor $D$.

Let $\bar{\eta}$ denote a geometric generic point of $\operatorname{Spec} \mathcal{O}_{\mathbf{P}, \bar{\xi}}$. We show that the character $\operatorname{det} H^{n}\left(X_{\bar{\eta}}, \mathbf{Q}_{\ell}\right)$ of $I_{\bar{\xi}}$ is the unique non-trivial character of order 2. By the result of [13] recalled in Lemma 2.10 .2 applied to $k=\overline{\mathbf{Q}}$, the geometric fiber $X_{\bar{\xi}}$ has a unique singular point which is an ordinary quadratic singularity in $X_{\bar{\xi}}$. Hence, by the Picard-Lefschetz formula [5, Théorème 3.4 (ii)], we have an exact sequence

$$
\begin{aligned}
0 \rightarrow & H^{n}\left(X_{\bar{\xi}}, \mathbf{Q}_{\ell}\right) \rightarrow H^{n}\left(X_{\bar{\eta}}, \mathbf{Q}_{\ell}\right) \rightarrow \mathbf{Q}_{\ell}\left(\frac{n}{2}\right) \\
& \rightarrow H^{n+1}\left(X_{\bar{\xi}}, \mathbf{Q}_{\ell}\right) \rightarrow H^{n+1}\left(X_{\bar{\eta}}, \mathbf{Q}_{\ell}\right) \rightarrow 0
\end{aligned}
$$

of $\ell$-adic representations of the inertia group $I_{\bar{\xi}}$. Further, since $X$ is regular, the base change $X_{\mathcal{O}_{\mathbf{P}, \bar{\xi}}}$ to the strict henselization is also regular and the inertia group $I_{\bar{\xi}}$ acts on $\mathbf{Q}_{\ell}\left(\frac{n}{2}\right)$ via the unique non-trivial character $I_{\bar{\xi}} \rightarrow\{ \pm 1\}$ by [5, Théorème 3.4 (iii)], [12]. Since $I_{\bar{\xi}}$ acts trivially on $H^{n+1}\left(X_{\bar{\xi}}, \mathbf{Q}_{\ell}\right)$ and on $H^{n}\left(X_{\bar{\xi}}, \mathbf{Q}_{\ell}\right)$, the boundary map $\mathbf{Q}_{\ell}\left(\frac{n}{2}\right) \rightarrow H^{n+1}\left(X_{\bar{\xi}}, \mathbf{Q}_{\ell}\right)$ in (3.7) is the zero-map and the character $\operatorname{det} H^{n}\left(X_{\bar{\eta}}, \mathbf{Q}_{\ell}\right)$ of $I_{\bar{\xi}}$ is non-trivial.

The composition map

$$
\Gamma\left(U_{\frac{1}{2}}, \mathcal{O}\right)^{\times} /\left(\Gamma\left(U_{\frac{1}{2}}, \mathcal{O}\right)^{\times}\right)^{2} \rightarrow H^{1}\left(U_{\frac{1}{2}}, \mathbf{Z} / 2 \mathbf{Z}\right) \rightarrow \operatorname{Hom}\left(I_{\bar{\xi}}, \mathbf{Z} / 2 \mathbf{Z}\right)
$$

is 0 since the strict henselization $\mathcal{O}_{\mathbf{P}, \bar{\xi}}$ contains $\overline{\mathbf{Q}}$ as a subfield. By (3.5) we thus get a map $\operatorname{Pic}\left(U_{\frac{1}{2}}\right)[2] \rightarrow \operatorname{Hom}\left(I_{\bar{\xi}}, \mathbf{Z} / 2 \mathbf{Z}\right)$.

Since the images of $\left[\operatorname{det} H^{n}(X)\right]$ and $\left[\operatorname{disc}_{d}(F)\right]$ in $\operatorname{Hom}\left(I_{\bar{\xi}}, \mathbf{Z} / 2 \mathbf{Z}\right)$ are non-trivial, the map $\operatorname{Pic}\left(U_{\frac{1}{2}}\right)[2] \rightarrow \operatorname{Hom}\left(I_{\bar{\xi}}, \mathbf{Z} / 2 \mathbf{Z}\right)$ is an isomorphism of groups of order 2 . Further by (3.5), the difference $\left[\operatorname{disc}_{d}(F)\right]-\left[\operatorname{det} H^{n}(X)\right]$ is in the image of the map

$$
\Gamma\left(U_{\frac{1}{2}}, \mathcal{O}\right)^{\times}=\mathbf{Z}\left[\frac{1}{2}\right]^{\times} \rightarrow H^{1}\left(U_{\frac{1}{2}}, \mathbf{Z} / 2 \mathbf{Z}\right) .
$$


Therefore, $\left[\operatorname{det} H^{n}(X)\right]$ equals either $\left[ \pm \operatorname{disc}_{d}(F)\right]$ or $\left[ \pm 2 \operatorname{disc}_{d}(F)\right]$. We show that the latter case is not possible. Let $K$ be the local field of $\mathbf{P}$ at the generic point of the fiber $\mathbf{P}_{\mathbf{F}_{2}}$. Then, the character [det $\left.H^{n}(X)\right]$ induces an unramified character of $\Gamma_{K}$. On the other hand, $\left[ \pm 2 \operatorname{disc}_{d}(F)\right]$ corresponds to a totally ramified quadratic extension of $K$. Hence the latter case is excluded and we obtain $\left[\operatorname{det} H^{n}(X)\right]=\left[ \pm \operatorname{disc}_{d}(F)\right]$.

It remains to show that the $\operatorname{sign}$ is $\varepsilon(n, d)$. To do so, let Spec $\mathbf{R} \rightarrow U$ be the map defined by the homogenous polynomial $f=T_{0}^{d}+\cdots+T_{n+1}^{d}$. Then, since $\operatorname{disc}_{d}(f)=d^{-a(n, d)} \operatorname{disc}_{r}(f)>0$, the pull-back of $\left[\operatorname{disc}_{d}(F)\right]$ in $H^{1}(\mathbf{R}, \mathbf{Z} / 2 \mathbf{Z})$ is 1 . On the other hand, by Corollary 1.3 and by Artin's comparison theorem relating singular and étale cohomology ([1, Théorème 4.1]), the pull-back of $\left[\operatorname{det} H^{n}(X)\right]$ equals $\varepsilon(n, d) \in H^{1}(\mathbf{R}, \mathbf{Z} / 2 \mathbf{Z})=\{ \pm 1\}$. Thus, the assertion is proved.

\section{Discriminant modulo 4}

In this section, we keep assuming that $n$ is even.

Let us first prove the following elementary fact:

Lemma 4.1. Let $K$ be a complete discrete valuation field such that 2 is a uniformizer. Let $u \in \mathcal{O}_{K}^{\times}$be a unit which is not a square and let $L$ denote the quadratic extension $K(\sqrt{u})$.

1. The extension $L$ is unramified over $K$ if and only if there exists a unit $v \in \mathcal{O}_{K}^{\times}$ such that $u \equiv v^{2}(\bmod 4)$.

2. Assume that the extension $L$ is unramified over $K$. Then, for every unit $v$ satisfying $u \equiv v^{2}(\bmod 2)$, we have $u \equiv v^{2}(\bmod 4)$. Further, the corresponding residue field extension is given by the Artin-Schreier equation $t^{2}+t=w$, where $w$ is the image of $\frac{1}{4}\left(u v^{-2}-1\right)$ in the residue field.

[Recall that "unramified" implies that the residue extension is separable.]

Proof. Let $F$ be the residue field of $K$. If $\bar{u} \in F^{\times}$is not a square, the residue field of $L$ is a quadratic radicial extension of $F$. Hence, by dividing $u$ by a square, we may assume $u \equiv 1(\bmod 2)$. We put $u=1+2 a$. Substituting $x=1+2 t$ into the equation $x^{2}=u$, we obtain $t^{2}+t=a / 2$. If $a$ is a unit, a solution $t$ cannot be an integer and we have $2 \operatorname{ord}_{L} t=-\operatorname{ord}_{L} 2$. Thus $L$ is totally ramified over $K$. If $a=2 b$, the extension $L$ is unramified and the residue extension is given by $t^{2}+t=\bar{b}$.

If $v^{2} \equiv v^{\prime 2}(\bmod 2)$, we have $\left(v / v^{\prime}\right)^{2} \equiv 1(\bmod 4)$ and assertion 2 follows.

Theorem 4.2. Let $n \geqslant 0$ and $d>1$ be integers. We assume that $n$ is even and define the sign $\varepsilon(n, d)= \pm 1$ by $(3.3)$. Then, $\varepsilon(n, d) \cdot \operatorname{disc}_{d}(F)$ is congruent to a square modulo 4 in $S^{m}\left(\left(S^{d} E\right)^{\vee}\right)=\Gamma(\mathbf{P}, \mathcal{O}(m))$, where $m=(n+2)(d-1)^{n+1}$. More precisely, there exist homogeneous polynomials $A \in S^{\frac{m}{2}}\left(\left(S^{d} E\right)^{\vee}\right)$ and $B \in S^{m}\left(\left(S^{d} E\right)^{\vee}\right)$ satisfying $\varepsilon(n, d) \cdot \operatorname{disc}_{d}(F)=A^{2}+4 B$.

Proof. Let $K$ be the local field of $\mathbf{P}$ at the generic point $\xi$ of the fiber $\mathbf{P}_{\mathbf{F}_{2}}$. Namely, $K$ is the fraction field of the completion of the local $\operatorname{ring} \mathcal{O}_{\mathbf{P}, \xi}$. The residue field $F=\kappa(\xi)$ is the function field of $\mathbf{P}_{\mathbf{F}_{2}}$. Take a global section $A_{1} \in \Gamma\left(\mathbf{P}, \mathcal{O}\left(\frac{m}{2}\right)\right)$ not divisible by 2 . The germ of $A_{1}$ is a basis of the stalk of $\mathcal{O}\left(\frac{m}{2}\right)$ at $\xi$. Since the germ of $\varepsilon(n, d) \cdot \operatorname{disc}_{d}(F)$ is also a basis of the stalk of $\mathcal{O}(m)$ at $\xi$, the ratio $\varepsilon(n, d) \cdot \operatorname{disc}_{d}(F) / A_{1}^{2}$ is a unit at $\xi$. 
By Theorem 3.5 we have

$$
\left[\operatorname{det} H^{n}(X)\right]=\left[\varepsilon(n, d) \cdot \operatorname{disc}_{d}(F)\right]
$$

in $H^{1}\left(U_{\mathbf{Z}\left[\frac{1}{2}\right]}, \mathbf{Z} / 2 \mathbf{Z}\right)$. Since $\left[\operatorname{det} H^{n}(X)\right]$ is the restriction of an element of $H^{1}(U, \mathbf{Z} / 2 \mathbf{Z})$, the extension of $K$ generated by the square root of $\varepsilon(n, d) \cdot \operatorname{disc}_{d}(F) / A_{1}^{2}$ is an unramified extension. Hence by Lemma 4.1.1, there exists a unit $v \in \mathcal{O}_{K}^{\times}$such that $\varepsilon(n, d) \cdot \operatorname{disc}_{d}(F) \equiv v^{2} \cdot A_{1}^{2}(\bmod 4)$.

We consider the local section $\bar{A}=v \cdot A_{1}(\bmod 2)$ of $\mathcal{O}_{\mathbf{P}_{\mathbf{F}_{2}}}(m)$. Since its square has no pole, the local section $\bar{A}$ has the same property and it defines a global section $\Gamma\left(\mathbf{P}_{\mathbf{F}_{2}}, \mathcal{O}(m)\right)$. Let us choose a lifting $A \in \Gamma(\mathbf{P}, \mathcal{O}(m))$ of $\bar{A}$. Since $\varepsilon(n, d)$. $\operatorname{disc}_{d}(F) / A^{2} \equiv 1(\bmod 2)$, we have $\varepsilon(n, d) \cdot \operatorname{disc}_{d}(F) / A^{2} \equiv 1(\bmod 4)$ by Lemma 4.1.2. Namely, the difference $\varepsilon(n, d) \cdot \operatorname{disc}_{d}(F)-A^{2}$ is divisible by 4 at $\xi$ and hence divisible on $\mathbf{P}$.

Corollary 4.3. Let $k$ be a field of characteristic 2 and $Y$ be a smooth hypersurface of even dimension $n$ in $\mathbf{P}_{k}^{n+1}$ defined by a homogeneous polynomial $f$ of degree $d$. Let $A(f) \in k^{\times}$and $B(f) \in k$ denote the specialization of the polynomials $A$ and $B$ occurring in Theorem 4.2.1. Then, the quadratic character $\operatorname{det} H^{n}\left(Y_{\bar{k}}, \mathbf{Q}_{\ell}\left(\frac{n}{2}\right)\right)$ of $\Gamma_{k}$ is defined by the Artin-Schreier equation $t^{2}+t=B(f) \cdot A(f)^{-2}$.

[In other words, the kernel of $\operatorname{det} V: \Gamma_{k} \rightarrow\{ \pm 1\}$ is the subgroup of $\Gamma_{k}$ corresponding to the Artin-Schreier extension of $k$ defined by $t^{2}+t=B(f) \cdot A(f)^{-2}$.]

Proof. Since the divided discriminant $\operatorname{disc}_{d}(F)$ is a basis of $\mathcal{O}_{U}(m)$, the section $A$ in Theorem 4.2 is a basis of $\mathcal{O}_{U_{\mathbf{F}_{2}}}\left(\frac{m}{2}\right)$. The pull-back of $\left[\operatorname{det} H^{n}(X)\right]$ in $H^{1}\left(U_{\mathbf{F}_{2}}, \mathbf{Z} / 2 \mathbf{Z}\right)$ is defined by $t^{2}+t=B \cdot A^{-2}$ by Theorem 4.2 and Lemma 4.1. Hence, the assertion follows by specialization.

\section{Examples of discriminants}

5.1. Binary forms. Let $F=C_{0} T_{0}^{d}+C_{1} T_{0}^{d-1} T_{1}+\cdots+C_{d} T_{1}^{d}$ be the universal binary polynomial of degree $d>1$ defining the universal family of finite schemes of degree $d$ in $\mathbf{P}^{1}$. The divided discriminant $\operatorname{disc}_{d}(F)$ is a homogeneous polynomial in $\left(C_{i}\right)$ of degree $m=2 d-2$ and the sign $\varepsilon(0, d)$ is $(-1)^{d(d-1) / 2}$.

The signed discriminant $\varepsilon(0, d) \cdot \operatorname{disc}_{d}(F)$ is equal to $\operatorname{dis}_{d}(F)=\tilde{\Delta}\left(C_{0}, \ldots, C_{d}\right)$ in the notation of [4, Chap. 4, Section $6, \mathrm{n}^{\mathrm{o}} 7$, formula (52)] where the subscript $d$ stands for the degree. For $d=2$, we have $\varepsilon(0,2)=-1$ and $\varepsilon(0,2) \cdot \operatorname{disc}_{d}(F)=b^{2}-4 a c$ for $(a, b, c)=\left(C_{0}, C_{1}, C_{2}\right)$.

The discriminant $\operatorname{disc}_{d}(F) \in \mathbf{Z}\left[C_{0}, \ldots, C_{d}\right]$ is the unique polynomial such that, if $F=\prod_{i=1}^{d}\left(u_{i} T_{0}-v_{i} T_{1}\right)$, then

$$
\operatorname{disc}_{d}(F)=\prod_{i \neq j}\left(u_{i} v_{j}-u_{j} v_{i}\right) .
$$

Indeed, if we invert $C_{0}$ and $u_{i}$ 's and if we put $s_{i}=C_{i} / C_{0}$ and $w_{i}=v_{i} / u_{i}$, we have an injection $\mathbf{Z}\left[C_{0}^{ \pm 1}, C_{1}, \ldots, C_{d}\right]=\mathbf{Z}\left[C_{0}^{ \pm 1}, s_{1}, \ldots, s_{d}\right] \rightarrow \mathbf{Z}\left[u_{1}^{ \pm 1}, \ldots, u_{d}^{ \pm 1}, v_{1}, \ldots, v_{d}\right]=$ $\mathbf{Z}\left[u_{1}^{ \pm 1}, \ldots, u_{d}^{ \pm 1}, w_{1}, \ldots, w_{d}\right]$ and the right-hand side is $C_{0}^{2(d-1)} \prod_{i \neq j}\left(w_{j}-w_{i}\right)$. Hence, the discriminant $\operatorname{disc}_{d}(F)$ is divisible in the polynomial ring $\mathbf{Z}\left[u_{1}, \ldots, u_{d}, v_{1}, \ldots, v_{d}\right]$ by the right-hand side and hence is a constant multiple of it since the degrees are 
equal. By evaluating at $u_{i}=1$ and $v_{i}=\zeta_{d}^{i}$ for a primitive $d$ th root $\zeta_{d}$ of 1 , we get $F=T_{0}^{d}-T_{1}^{d}$ and $d^{d-2} \cdot \operatorname{disc}_{d} F=\operatorname{disc}_{r} F=\operatorname{res}\left(d T_{0}^{d-1},-d T_{1}^{d-1}\right)=(-1)^{d-1} d^{2(d-1)}$. For the right-hand side, we have $\prod_{i \neq j}\left(\zeta_{d}^{j}-\zeta_{d}^{i}\right)=\zeta_{d}^{\left(\begin{array}{c}d \\ 2\end{array}\right)(d-1)} \prod_{i=1}^{d-1}\left(1-\zeta_{d}^{i}\right)^{d}=(-1)^{d-1} d^{d}$ and (5.1) follows.

Hence, the signed discriminant is given by

$$
(-1)^{d(d-1) / 2} \cdot \operatorname{disc}_{d}(F)=\prod_{i<j}\left(u_{i} v_{j}-u_{j} v_{i}\right)^{2}
$$

it is congruent to the square of $\prod_{i<j}\left(u_{i} v_{j}+u_{j} v_{i}\right)$ in $\mathbf{Z}\left[C_{0}, \ldots, C_{d}\right]$ modulo 4 . Note that $\prod_{i<j}\left(u_{i} v_{j}+u_{j} v_{i}\right)=C_{0}^{2(d-1)} \prod_{i<j}\left(w_{i}+w_{j}\right)$ is a symmetric polynomial in $w_{i}$ 's and hence is a polynomial in $\mathbf{Z}\left[C_{0}, \ldots, C_{d}\right] \subset \mathbf{Z}\left[C_{0}^{ \pm 1}, s_{1}, \ldots, s_{d}\right]$.

5.2. Quadrics. Let $F=\sum_{0 \leqslant i \leqslant j \leqslant n+1} C_{i j} T_{i} T_{j}$ be the universal quadratic polynomial defining the universal family of quadrics of even dimension $n \geqslant 0$. The divided discriminant $\operatorname{disc}_{d}(F)$ is the same as the resultant discriminant $\operatorname{disc}_{r}(F)$; it is a homogeneous polynomial in $\left(C_{i j}\right)$ of degree $m=n+2$ and the sign $\varepsilon(n, 2)$ is $(-1)^{(n+2) / 2}$.

Let $A=\left(A_{i j}\right)$ be the $m \times m$ symmetric matrix with coefficients in $\mathbf{Z}\left[C_{i j} ; 0 \leqslant i \leqslant\right.$ $j \leqslant n+1]$ defined by $A_{i j}=A_{j i}=C_{i j}$ for $i<j$ and $A_{i i}=2 C_{i i}$. We have $T A^{t} T=2 F$ where $T$ is the row vector $\left(T_{0}, \ldots, T_{n+1}\right)$ and

$$
\operatorname{disc}_{d}(F)=\operatorname{disc}_{r}(F)=\operatorname{det} A .
$$

Indeed, by inverting 2 , we see that $\operatorname{det} A$ equals the divided $\operatorname{discriminant} \operatorname{disc}_{d}(F)$ up to a constant $\lambda$ which is \pm a power of 2 . For the unit quadratic form $\sum_{i=0}^{n+1} T_{i}^{2}$, we have $\operatorname{disc}_{r}(F)=2^{n+2}=\operatorname{det} A$ hence $\lambda=1$. This proves (5.3).

Let $k$ be a field of characteristic $\neq 2$ and let $Q$ be a smooth quadric of even dimension $n$ in $\mathbf{P}_{k}^{n+1}$ defined by a non-degenerate quadratic form $q(x)={ }^{t} x B x$ for a symmetric matrix $B \in M_{n+2}(k)$. Then, the character $\operatorname{det} H^{n}\left(Q_{\bar{k}}, \mathbf{Q}_{\ell}\left(\frac{n}{2}\right)\right)$ is defined by the square root of the signed discriminant $\varepsilon(n, 2) \operatorname{disc}_{d} q=(-1)^{\frac{n+2}{2}} 2^{n+2} \operatorname{det} B$; this follows from (5.3) applied to $A=2 B$.

5.3. Plane cubics. Let $C \rightarrow \mathbf{P}=\mathbf{P}\left(\left(S^{3} E\right)^{\vee}\right)$ be the universal family of cubic curves defined by the universal cubic polynomial $F$ for $n=1$ and $d=3$. The divided discriminant $\operatorname{disc}_{d}(F)$ may be viewed as a section of $\Gamma(\mathbf{P}, \mathcal{O}(12))$. We compare it with the discriminant of the Jacobian defined by a Weierstrass equation.

Let $p: \mathbb{P} \rightarrow \mathbf{P}$ denote the $\mathbf{P}^{2}$-bundle $\mathbf{P}\left(\mathcal{E}^{\vee}\right)=\operatorname{Proj} S^{\bullet} \mathcal{E}^{\vee}$ defined by the dual of $\mathcal{E}=\mathcal{O}(2) \oplus \mathcal{O}(3) \oplus \mathcal{O}$ and let $X \in \Gamma\left(\mathbb{P}, p^{*} \mathcal{O}(2) \otimes \mathcal{O}(1)\right), Y \in \Gamma\left(\mathbb{P}, p^{*} \mathcal{O}(3) \otimes \mathcal{O}(1)\right), Z \in$ $\Gamma(\mathbb{P}, \mathcal{O}(1))$ denote the components of the tautological map $p^{*} \mathcal{E}^{\vee} \rightarrow \mathcal{O}(1)$. For $i=$ $1,2,3,4,6$, homogeneous polynomials $a_{i}(F) \in S^{i}\left(S^{3} E\right)^{\vee}=\Gamma(\mathbf{P}, \mathcal{O}(i))$ of degree $i$ of the coefficients of $F$ are defined in $[2,(1.6)]$. Let $W$ be the closed subscheme of $\mathbb{P}$ defined by the homogeneous Weierstrass equation

$$
E: Y^{2} Z+a_{1} X Y Z+a_{3} Y Z^{2}-\left(X^{3}+a_{2} X^{2} Z+a_{4} X Z^{2}+a_{6} Z^{3}\right)=0 .
$$

Then the functor $\mathrm{Pic}_{C / \mathbf{P}}^{0}$ is represented by the smooth locus $J$ of $W$ [2, Theorem 1 . Let $\operatorname{disc}_{e}(E) \in \Gamma(\mathbf{P}, \mathcal{O}(12))$ denote the discriminant of the Weierstrass equation (5.4) in the standard sense defined as a polynomial of $a_{i}(F)$, cf. [2, (1.3)]. Then, we 
have

$$
\operatorname{disc}_{d}(F)=-\operatorname{disc}_{e}(E) .
$$

Indeed, since both $\operatorname{disc}_{d}(F)$ and $\operatorname{disc}_{e}(E)$ are bases on the smooth locus $U=\mathbf{P}-D$ and $\Gamma\left(U, \mathcal{O}^{\times}\right)=\mathbf{Z}^{\times}=\{ \pm 1\}$, we have $\operatorname{disc}_{d}(F)= \pm \operatorname{disc}_{e}(E)$. We determine the sign by testing it on the Fermat cubic $f=x^{3}+y^{3}+z^{3}$. By $(2.1)$, we have $\operatorname{disc}_{d}(f)=$ $3^{-3} \operatorname{disc}_{r}(f)=3^{-3} \cdot 3^{4 \cdot 3}=3^{9}$. On the other hand, since $c_{4}$ and $c_{6}([2,(1.3)])$ are given by $c_{4}=0$ and $c_{6}=2^{3} \cdot 3^{6}$, we obtain $\operatorname{disc}_{e}(E)=-3^{9}$.

5.4. Cubic surfaces. We put $A=\mathbf{Z}[a, b, c, d, e]$ and $\mathbb{P}=$ Proj $A$. Define a closed subscheme $S$ of $\mathbf{P}^{4} \times \mathbb{P}=\operatorname{Proj} \mathbf{Z}[x, y, z, u, v] \times \mathbb{P}$ by the Sylvester-type equations

$$
x+y+z+u+v=0, a x^{3}+b y^{3}+c z^{3}+d u^{3}+e v^{3}=0 .
$$

It is known that a general enough cubic equation in characteristic 0 can be put in that form after a suitable finite extension of the ground field; cf. [7, Section 9.4.1]. In 1862, Salmon ([15, Section 543]) showed that, over C, the corresponding cubic surface is smooth if and only if a certain polynomial $\operatorname{disc}_{s}(a, b, c, d, e) \in A$ is non-zero. This polynomial is homogeneous of degree 32 in $a, b, c, d, e$. Its definition is:

$$
\operatorname{disc}_{s}(a, b, c, d, e)=\left(\left(s^{2}-64 r t\right)^{2}-4 t^{3} p\right)^{2}-2^{11}\left(8 t^{6} q+t^{4} s\left(s^{2}-4 r t\right)\right),
$$

where $p=a+b+c+d+e, q=a b+\cdots, r=a b c+\cdots, s=a b c d+\cdots, t=a b c d e$, are the elementary symmetric functions of $a, b, c, d, e$. [We give here the corrected version of the formula due to W.L. Edge, cf. [8] and [9]; there were numerical mistakes in [15].]

By eliminating one variable in (5.6), one obtains a cubic polynomial $F_{s}$ with coefficients in $A$ and its divided discriminant $\operatorname{disc}_{d}\left(F_{s}\right)$ is a well-defined element of $A$. The relation between the Salmon discriminant and the divided one is:

$$
\operatorname{disc}_{s}(a, b, c, d, e)=3^{-27} \operatorname{disc}_{d}\left(F_{s}\right) .
$$

Indeed, the polynomial $\operatorname{disc}_{s}(a, b, c, d, e)$ is geometrically irreducible [9, Lemma 2.5] and the smooth locus of $S_{\mathbf{Q}} \rightarrow \mathbb{P}_{\mathbf{Q}}$ is defined by $\operatorname{disc}_{s}(a, b, c, d, e) \neq 0$, cf. [15], loc.cit., and $\left[9\right.$, Corollary 2.10]. This implies that $\operatorname{disc}_{s}(a, b, c, d, e)$ is a constant multiple of $\operatorname{disc}_{d}\left(F_{s}\right)$. We determine the constant by testing it on the Fermat cubic

$$
f=y^{3}+z^{3}+u^{3}+v^{3}
$$

corresponding to $(a, b, c, d, e)=(0,1,1,1,1)$.

By $(5.7)$, we have $\operatorname{disc}_{s}(0,1,1,1,1)=1$. On the other hand, we have $\operatorname{disc}_{d}(f)=$ $3^{-5} \operatorname{disc}_{r}(f)=3^{-5} 3^{32}=3^{27}$. This proves formula (5.8).

For the Clebsch surface corresponding to $(a, b, c, d, e)=(1,1,1,1,1)$, we have $\operatorname{disc}_{s}(1,1,1,1,1)=-3^{5} 5$ and the equality (5.8) implies $\operatorname{disc}_{d}(f)=-3^{32} 5$ for the corresponding polynomial $f=\sum x^{3}-\left(\sum x\right)^{3}$. Since $f$ is divisible by 3 , this means that $\operatorname{disc}_{d}(f / 3)=-5$, which fits with the fact that $f / 3$ defines a smooth cubic in every characteristic except 5 . See also [9, Remark 2.11].

Let $S \subset \mathbf{P}_{k}^{3}$ be a smooth cubic surface defined by a cubic form $f \in S^{3} E_{k}$. Then $H^{2}\left(S_{\bar{k}}, \mathbf{Q}_{\ell}(1)\right)$ is spanned by the classes of the 27 lines [18, p. 588], [14, Section 27]. The group of automorphisms of the $\mathbf{Z}$-lattice spanned by the classes of these lines permuting them and preserving the intersection form is isomorphic to the Weyl group 
$W\left(E_{6}\right)$ of the root system $E_{6}$. The kernel of the determinant map $W\left(E_{6}\right) \rightarrow\{ \pm 1\}$ is a simple group of order 25920 .

The action of $\Gamma_{k}$ on the 27 lines defines a homomorphism

$$
\Gamma_{k} \rightarrow W\left(E_{6}\right)
$$

unique up to conjugation. By applying Theorem 3.5 and using specialization, we see that the composition $\Gamma_{k} \rightarrow W\left(E_{6}\right) \rightarrow\{ \pm 1\}$ is defined by the square root of the signed discriminant $-\operatorname{disc}_{d} f$. Note that for a cubic surface, we have $n=2, d=3$ so that $\varepsilon(n, d)=-1$. In the case where the characteristic of $k$ is not 3 , this is equivalent to $\left[9\right.$, Theorem 2.12] which is stated in terms of the Salmon discriminant $\operatorname{disc}_{s} f$.

The formula (5.7) together with (5.8) and $\varepsilon(2,3)=-1$ implies the congruence $\varepsilon(2,3) \operatorname{disc}_{d}\left(F_{s}\right) \equiv-3 s^{4}(\bmod 8)$. Consequently, for a cubic surface in Sylvester form (5.6) in characteristic 2, the determinant map $\Gamma_{k} \rightarrow\{ \pm 1\}$ is trivial if and only if $k$ contains $\mathbf{F}_{4}$.

\section{Acknowledgments}

The author would like to express his sincere gratitude to Jean-Pierre Serre for asking the author to compute the determinant in terms of the discriminant of a defining polynomial. He kindly allows the author to include his topological argument in Section 1 and Examples on cubic curves and cubic surfaces in Section 5. The author thanks Serre and an anonymous referee for numerous and helpful comments to improve the presentation of the article. The author also thanks Michel Demazure for showing him a manuscript of [6] before publication. The research is partially supported by JSPS Grant-in-Aid (A) 22244001.

\section{References}

[1] M. Artin, Théorème de changement de base par un morphisme lisse et applications, SGA 4 III Exposé XVI, Springer Lecture Notes in Math. 305, 209-252 (1973).

[2] M. Artin, F. Rodriguez-Villegas and J. Tate, On the Jacobians of plane cubics, Adv. Math. 198 (2005), 366-382.

[3] J. Bochnak, M. Coste and M-F. Roy, Géométrie Algébrique Réelle, Springer-Verlag, 1987; revised English translation, Real Algebraic Geometry, Ergebn. Math. (3) 36, Springer-Verlag, 1998.

[4] N. Bourbaki, Algèbre - Chapitres 4 à 7, Paris, Masson, 1981; new printing, Springer-Verlag, 2006; English translation, Algebra II, Springer-Verlag, 1989.

[5] P. Deligne, La formule de Picard-Lefschetz, SGA 7 II Exposé XV, Springer Lecture Notes in Math. 340 (1973), 165-196.

[6] M. Demazure, Résultant, Discriminant, unpublished Bourbaki manuscript dated July 1969; to appear in L'Enseignement Mathématique.

[7] I.G. Dolgachev, Topics in classical algebraic geometry, Part I, Lecture Notes, University of Michigan, 2007.

[8] W.L. Edge, The discriminant of a cubic surface, Proc. R. Irish Acad., 80 (1980), 75-78.

[9] A.-S. Elsenhans and J. Jahnel, The discriminant of a cubic surface, http://arxiv.org/abs/ 1006.0721

[10] I.M. Gelfand, M.M. Kapranov and A. Zelevinsky, Discriminants, Resultants, and Multidimensional Determinants, Birkhäuser, Boston, 1994.

[11] A. Grothendieck, Éléments de Géométrie Algébrique, III (Première Partie), Publ. Math. IHES 11, (1961).

[12] L. Illusie, Sur la formule de Picard-Lefschetz, Algebraic Geometry 2000, Azumino (Hotaka), Adv. Stud. Pure Math., 36 (2002), 249-268. 
[13] N.M. Katz, Pinceaux de Lefschetz: Théorème d'existence, SGA 7 II Exposé XVII, Springer Lecture Notes in Math. 340 (1973), 212-253.

[14] Y.I. Manin, Cubic forms: algebra, geometry, arithmetic, 2nd edn., North-Holland, Amsterdam, 1986.

[15] G. Salmon, A Treatise on the analytic geometry of three dimensions, Dublin, 1862; 5th edn., Dublin, 1915; French translation, Gauthier-Villars, 1882.

[16] J-P. Serre, Zeta and L-functions, Arithmetical Algebraic Geometry, Harper and Row, New York (1965), 82-92, (Oe. 64, volume 2, 249-259).

[17] J-P. Serre, Lectures on $N_{X}(p)$, (Research Notes in Mathematics) A K Peters/CRC Press (2012).

[18] A. Weil, Abstract versus classical algebraic geometry, Proc. ICM Amsterdam (1954), vol. III, 550-558 (= Coll. Papers, vol. II, [1954h]).

Department of Mathematical Sciences, University of Tokyo, Tokyo 153-8914, Japan

E-mail address: t-saito@ms.u-tokyo.ac.jp 
